\title{
Primary Subcapsular Reflux as an Etiology of Subcapsular Renal Abscess
}

\author{
Eun Ju Yoo, M.D. ${ }^{1}$ \\ Jae Hyuk Oh, M.D. ${ }^{1}$ \\ Hyun Joo Jung, M.D., Ph.D. ${ }^{1}$ \\ Su Jin Lee, M.D., Ph.D. ${ }^{2}$ \\ Ji Eun Park, M.D., Ph.D. ${ }^{3}$ \\ Ki Soo Pai, M.D., Ph.D. ${ }^{1}$ \\ ${ }^{1}$ Departments of Pediatrics, ${ }^{2}$ Nuclear \\ Medicine, and ${ }^{3}$ Radiology, Ajou \\ University School of Medicine, Suwon, \\ Republic of Korea
}

Corresponding author:

Ki Soo Pai, M.D., Ph.D.

Department of Pediatrics, Ajou University School of Medicine, 164, World cup-ro, Yeongtong-gu, Suwon 16499, Republic of Korea

Tel: +82-31-219-5160

Fax: +82-31-219-5169

E-mail:kisoopai@hanmail.net

Received: 21 October 2021

Revised: 30 October 2021

Accepted: 8 November 2021
This is an open-access article distributed under the terms of the Creative Commons Attribution Non-Commercial License (http:// creativecommons.org/licenses/by-nc/4.0/) which permits unrestricted non-commercial use, distribution, and reproduction in any medium, provided the original work is properly cited.

Copyright (C) 2021 The Korean Society of Pediatric Nephrology
Herein, we report two rare cases of renal infection. The first case was renal subcapsular urine reflux in a 8-month-old girl with recurrent urinary tract infection and the second was subcapsular abscess in a 14-year-old girl with diabetes, who was successfully treated with percutaneous drainage. It has been suggested that renal subcapsular abscesses could be caused by the direct reflux of urine into the subcapsular space, rather than spread of infection from an existing parenchymal lesion, and that complete recovery can be achieved if percutaneous drainage is performed in a timely manner. We propose primary subcapsular reflux, in which urine directly refluxes upwards into the subcapsular space of the kidney, as one of the mechanisms for development of renal subcapsular abscesses.

Key words: Abcess, Drainage, Urinary tract infections, Vesico-ureteral reflux

\section{Introduction}

Renal and perirenal abscesses are the most severe forms of kidney infection, in which abscesses are formed within the renal parenchyma or in the fat layer between the renal capsule and renal fascia. Besides, subcapsular renal abscess is a very rare type of renal abscess, which is defined by a suppurative process localized to the space between the renal capsule and the renal parenchyma ${ }^{1)}$.

The diagnosis of renal abscess was greatly facilitated by the introduction of ultrasonography (US) and computed tomography (CT) into clinical practice in the 1970s, and a complete cure of the condition without surgery was made possible by treatment with percutaneous drainage (PCD) and broad-spectrum antibiotics. However, the diagnosis and treatment of renal and perirenal abscesses is often delayed in children because of nonspecific symptoms. Experience with successful treatment of kidney abscesses was first reported in adult patients in Korea in the $1980 \mathrm{~s}^{2)}$ and was followed by in children in 1985 by Choi et al. ${ }^{3)}$ and in 2014 by Shim et al. ${ }^{4}$; however, there have been no report regarding the treatment of subcapsular renal abscesses in children.

\section{Case report}

\section{Case 1: Renal Subcapsular Reflux}

An 8-month-old girl visited our hospital because of repeated urinary tract 
infection (UTI) and abnormal US findings of left-sided mild pelviectasis and a thickened right renal capsule. The infant had been delivered at full term with no specific perinatal problems, but had two febrile UTIs at 3 months and 4 months of age. For both UTIs, third-generation cephalosporin antibiotics were administered for 2 weeks. An US examination performed on May 7, 2018 at the time of the first febrile UTI revealed no abnormal findings (Fig. 1A); however, US performed 5 weeks later (June 15) due to recurrent infection showed thickening of the right renal capsule (Fig. 1B). Three months later, the patient visited our hospital for further evaluation and management. A Tc-99m dimercaptosuccinic acid (DMSA) scan performed on September 27, 2018 was normal; however, voiding cystourethrography (VCUG) performed on October 5, 2018 revealed grade 2 reflux in the right kidney, along with a rare phenomenon in which the contrast medium extended to the renal capsule and enveloping the entire kidney (Fig. 2). No specific treatment or antibiotic prophylaxis was administered to the patient, except for parental education regarding the patient's perineal hygiene. No additional renal infection has developed during the past 3 years. The patient's latest US examination on May 24, 2021 showed normal findings and the length measurements of right and left kidneys were $5.33 \mathrm{~cm}$ and $5.25 \mathrm{~cm}$, respectively (Fig. 1C).

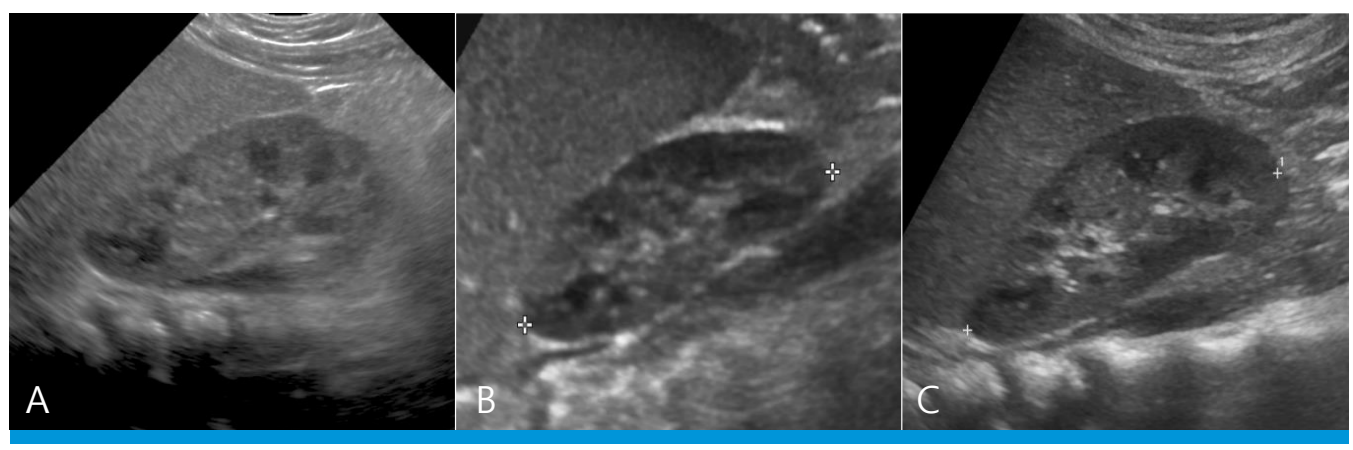

Fig. 1. Serial ultrasound examinations of the right kidney on May 7, 2018 (A), 6 weeks later on June 15 due to recurrent infection (B), and 3 years later on May 24, 2021 (C).

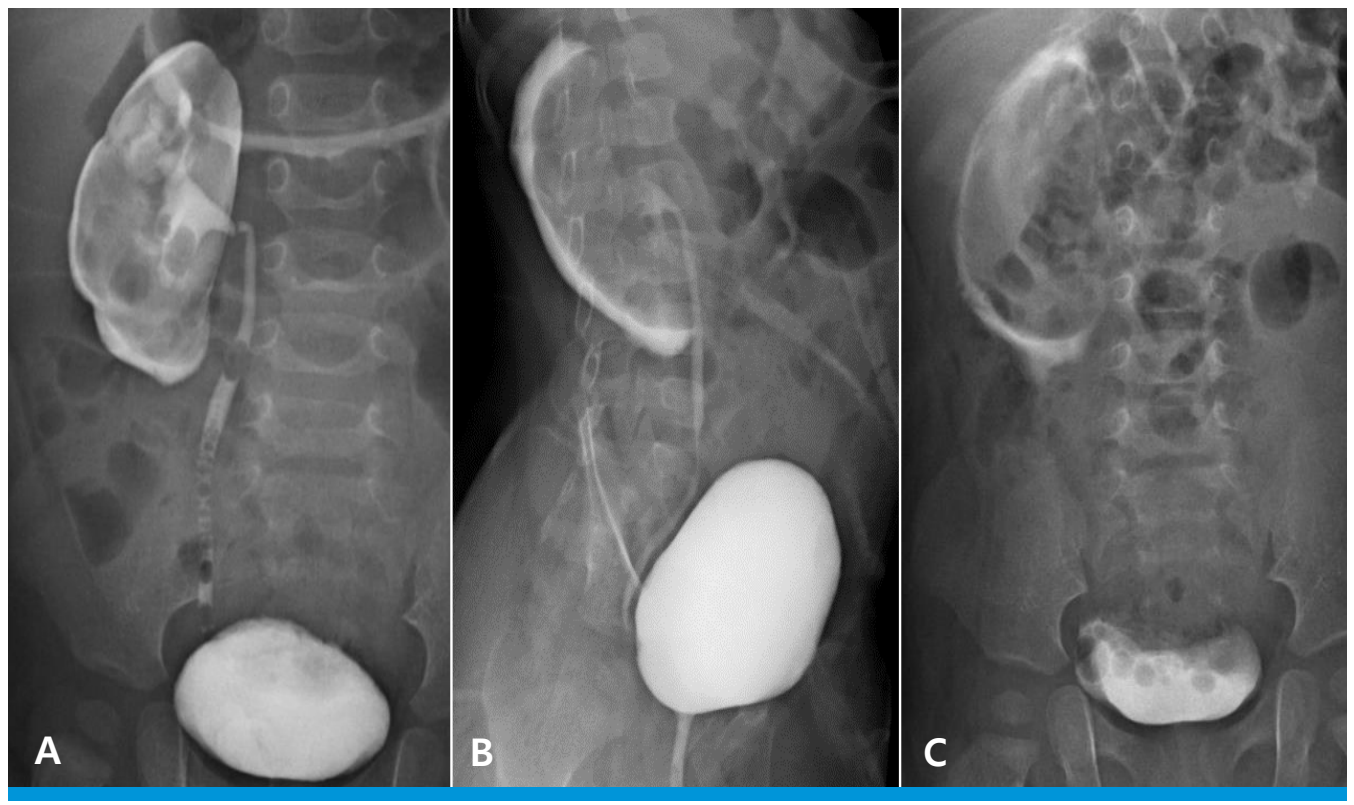

Fig. 2. Voiding cystourethrogram taken on October 5, 2018 showed grade II reflux, with leak of contrast medium to the entire subscapsular space of right kidney (A), and this was proved as subcapsular collection on the lateral view (B). There was no evidence of extrarenal leakage until urination was completed during examination (C). 


\section{Case 2: Renal Subcapsular Abscess}

A 14-year-old girl, in previous good health with no specific medical history after being delivered at 39 weeks of gestational age with a body weight of $3,500 \mathrm{~g}$, was admitted to the hospital with a fever of duration of 8 days and right flank pain. The patient was diagnosed with renal abscesses of the right kidney on abdominal CT. Intravenous antibiotic treatment for 7 days relieved the patient's clinical symptoms; however, follow-up CT revealed that the size of the abscesses had increased significantly. Furthermore, the patient was newly diagnosed with type 2 diabetes and was referred to our hospital for proper evaluation and treatment. Upon admission, the patient was alert and physical examination findings were: pulse rate, 85 beats/min; respiration rate, 20 breaths $/ \mathrm{min}$; body temperature, $36.9^{\circ} \mathrm{C}$; blood pressure, 130/95 mmHg; body weight, $88 \mathrm{~kg}$ (100th percentile); height, $163 \mathrm{~cm}$ (75th percentile); BMI, 33.12 $\mathrm{kg} / \mathrm{m}^{2}$; and no other findings except tenderness on the right kidney angle.
At the time of first admission to the referral hospital on January 25, 2021 blood tests revealed the followings: WBC

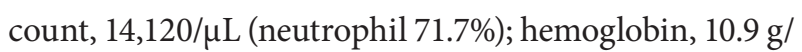
dL; hematocrit, 34.6\%; platelet count, 302,000/ $\mathrm{L} ; \mathrm{ESR}, 71$ $\mathrm{mm} / \mathrm{hr}$; CRP, $16.26 \mathrm{mg} / \mathrm{dL}$; and BUN/Cr, 9.1/0.47 mg/dL. Urine test results were as follows: $\mathrm{pH}, 6.0$; WBC, 10-29/ HPF; and RBC, 5-9/HPF. Later, Klebsiella pneumoniae grew to more than $10^{5} \mathrm{CFU} / \mathrm{ml}$ from urine culture.

Laboratory tests performed at our hospital on February 1, 2021 showed leukocyte count, 8,700/ $\mu \mathrm{L}$ (neutrophil 61.0 \%); hemoglobin, $9.9 \mathrm{~g} / \mathrm{dL}$; hematocrit, 31.6\%; platelet count,

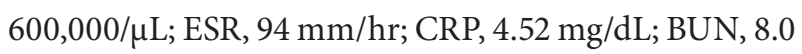
$\mathrm{mg} / \mathrm{dL}$; and $\mathrm{Cr}, 0.45 \mathrm{mg} / \mathrm{dL}$. Urine examination showed no pyuria or hematuria, and no pathogens were cultured. However, a small amount of non ESBL-producing Klebsiella pneumoniae continued to grow from the pus initially drained by PCD on the second day at our hospital.

The initial CT performed at the referral hospital on January 25,2021 revealed two abscess lesions in the upper and

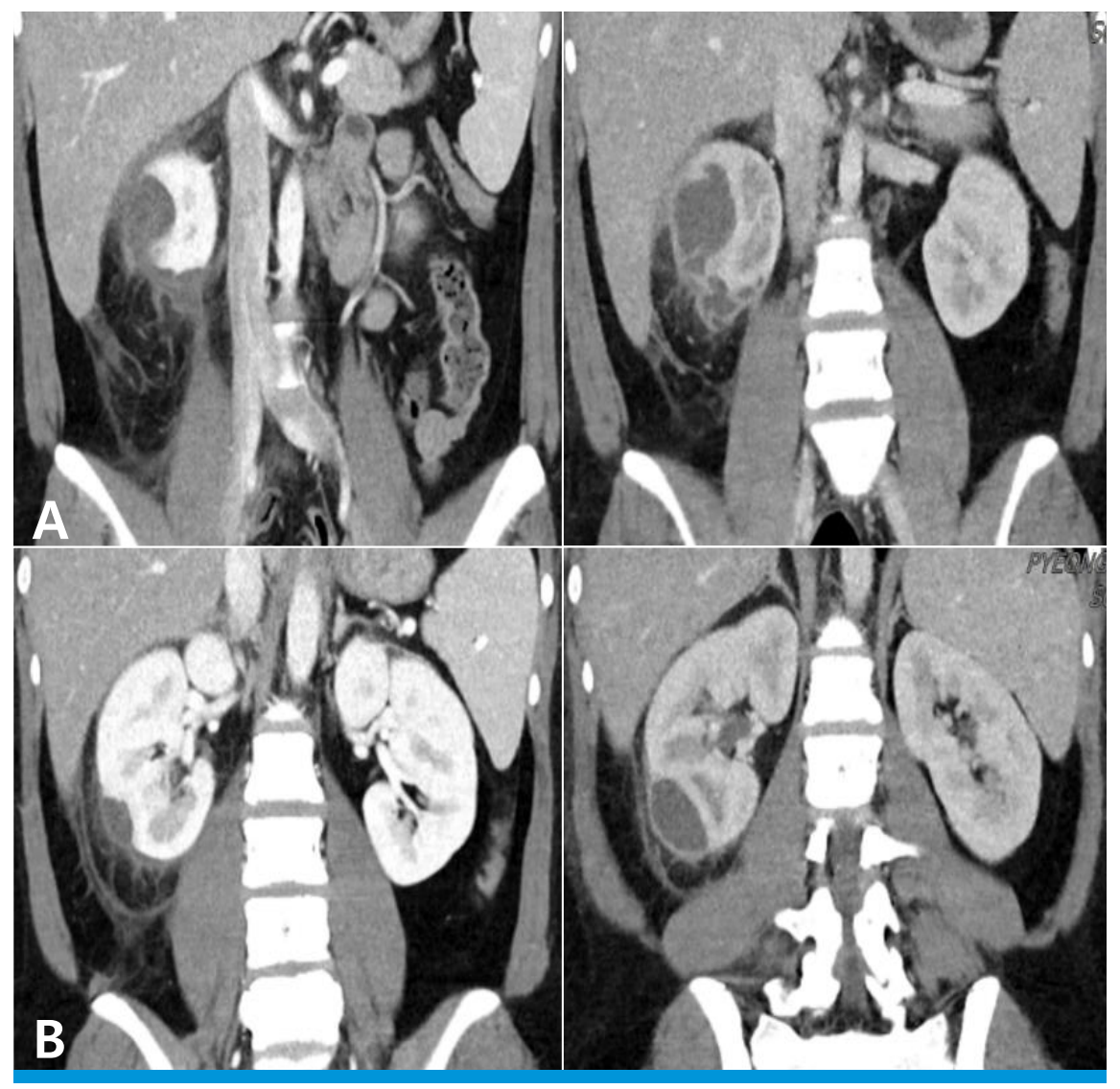

Fig. 3. Renal computed tomography on January 25, 2021 showed abscess formation in the upper and lower portion of right kidney $(A)$ and the follow-up exam on February 1, 2021 revealed that the size of abscesses had expanded (B). 
lower poles of the right kidney that were $2.0 \mathrm{~cm}$ and $1.0 \mathrm{~cm}$ in length, respectively (Fig. 3A). On the follow-up CT on February 1, 2021, 7 days after antibiotic treatment, the abscesses had increased in diameter to 4.5 and $2.0 \mathrm{~cm}$, respectively (Fig. 3B), and the patient was transferred to our hospital.

On arrival at our hospital, we reviewed the previous CT scans and confirmed the subcapsular positions of the abscesses, based on the findings that the renal cortex was compressed by the pus pockets, but still preserved. Therefore, an 8.5 French catheter was inserted to drain the pus (Fig. 4A) and a VCUG study performed 3 days later revealed no sign of reflux (Fig. 4B).

On renal US performed on January 25, 2021, the sizes of the right and left kidneys were found to be $11.8 \mathrm{~cm}$ and $11.2 \mathrm{~cm}$, respectively (The average kidney size of Korean female adolescents is as follows; right $9.68 \mathrm{~cm}$, left 9.96 $\mathrm{cm})$. The findings indicated the presence of an abscess at the lower pole of the right kidney, measuring $2.0 \mathrm{~cm}$ in diameter; another abscess was seen at the upper pole (Fig. 4C-1). Sequential US studies showed regression in the size of the renal abscesses compared to their initial appearances on January 25, 2021. Four days after PCD, US showed only the catheter in cross-section, with no evidence of the abscesses (Fig. 4C-2); the measured sizes of the right and left kidneys were $12.7 \mathrm{~cm}$ and $12.0 \mathrm{~cm}$, respectively. This renal enlargements in the absence of any abscess suggest that parenchymal in flammation still remains. The catheter was removed 5 days later. The final US performed 3 weeks later on February 24, 2021, showed that there was no abscess and both kidneys returned to normal in shape and size (right: $11.0 \mathrm{~cm}$, left: $11.5 \mathrm{~cm})$ (Fig. 4C-3).

For the treatment intravenous $(I V)$ cefotaxime was started on January 25, 2021 and $I V$ amikacin was added on January 28, 2021 in the previous hospital. Since we do not know the results of urine culture test at the previous

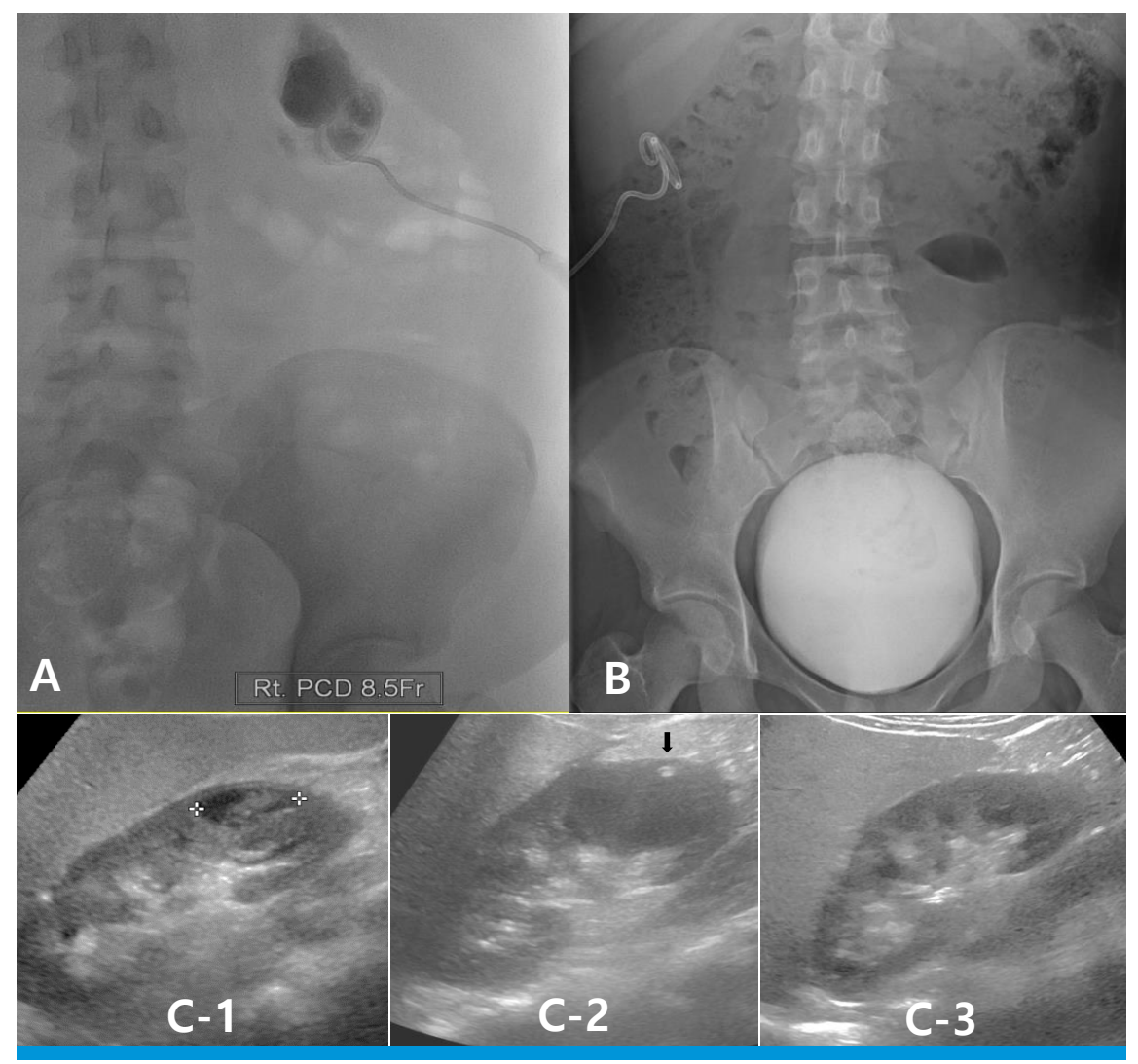

Fig. 4. A percutaneous drainage catheter was inserted into the subcapsular pocket to drain pus on February 2, 2021 (A) and VCUG was performed on 3 days after admission with no evidence of reflux stream (B). Renal ultrasonography on January 25, 2021 showed an abscess at the lower pole of the right kidney $(C-1)$. This lesion disappeared 4 days after PCD, and the PCD catheter tip was noted (arrow) (C-2). Follow-up examination on February 24, 2021 showed complete recovery of the right kidney $(C-3)$. 
hospital, we only added $\mathrm{PCD}$ procedure and did not change the antibiotics, to cover potential gram-negative bacteria and resistant ones, which are common causes of abscesses.

Using the PCD procedure, $35 \mathrm{~mL}$ of pus was drained during the first 8 hours, and then $5-14 \mathrm{~mL}$ for the next days. Complete reduction in the size of the abscess was confirmed on the 4th day, and the PCD catheter was locked and removed on the 7th day. On the 9th day after admission, the patient was discharged from the hospital with oral antibiotics (cefixime). In total, third generation cephalosporin antibiotics was administered for 15 days and $I V$ amikacin for 12 days. We did not quantitate the amikacin level since the serum Cr level was $0.47 \mathrm{mg} / \mathrm{dL}$ (normal range: 0.3-0.7 $\mathrm{mg} / \mathrm{dL}$ ) on the 1st day of hospitalization and $0.52 \mathrm{mg} / \mathrm{dL}$ on the 6 th day of hospitalization. For the next 6 months, there was no recurrence of UTI and the latest DMSA scan on August 18, 2021 showed full recovery from the large photon defect in the lower pole of the right kidney compared to the initial scan on February 3, 2021 (Fig. 5).

\section{Discussion}

Abscesses of the kidney and surrounding structures are named for its anatomic locations and is classified into renal parenchymal abscess, subcapsular renal abscess, perirenal abscess, or pararenal (extra renal) abscess (Fig. 6). Rupture of the renal capsule following exacerbation of a parenchymal abscess can lead to formation of a perirenal abscess that is confined to the fat layers surrounding the kidney while pararenal abscess is created by further extension of perirenal abscess or is secondary to the independent infections of adjacent organs.

Renal abscesses are rare but serious diseases that can cause severe renal damage. Luis et al. reported that the most common symptoms of renal abscesses in children were fever, abdominal pain, and other nonspecific symptoms such as nausea, vomiting, general weakness, and decreased appetite ${ }^{5}$. Due to these non-specific symptoms of renal abscesses, diagnosis and treatment are usually delayed by

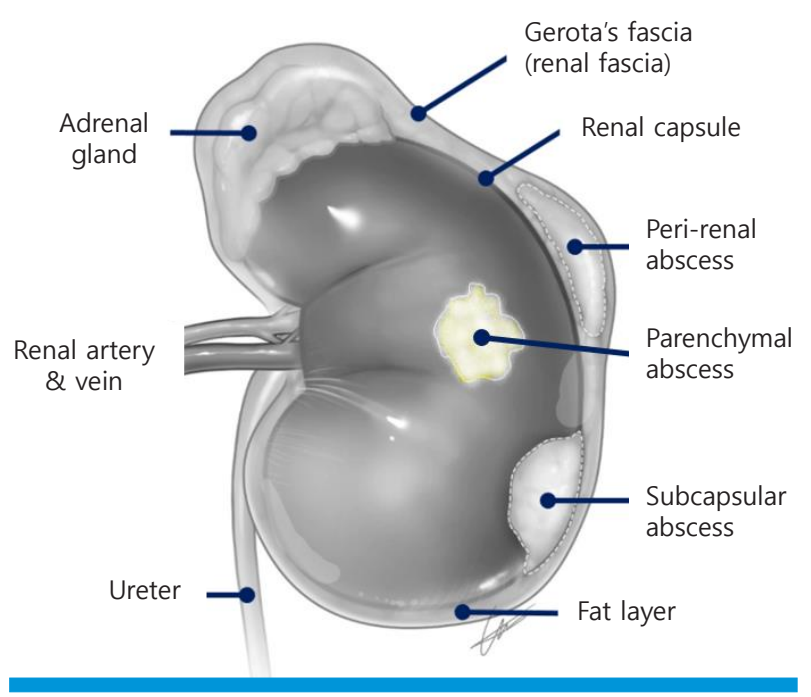

Fig. 6. Classification of renal abscesses: picture produced by the authors to facilitate understanding of the differential diagnosis of renal abscesses based on their locations.

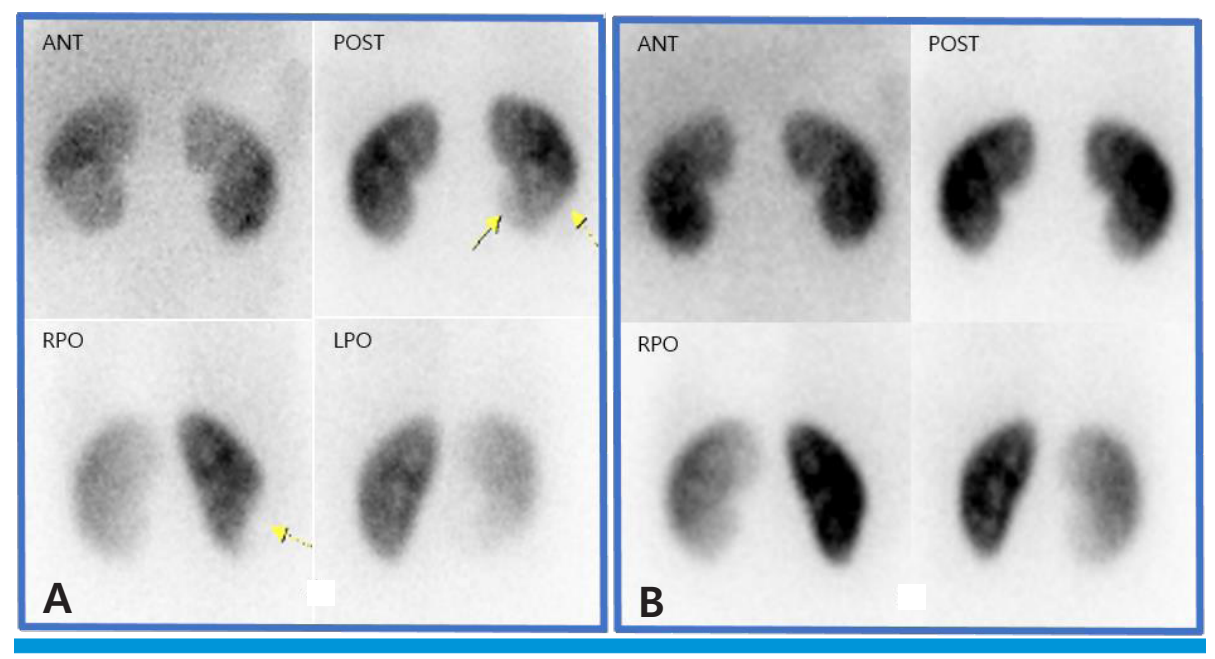

Fig. 5. Tc-99m DMSA renal scan on February 3, 2021 showed large photon defect in lower portion of right kidney $(A)$, and the lesion completely disappeared on the follow-up exam after 6 months (B). 
a week ${ }^{6}$.

Until the 1950s, most renal abscesses developed as the result of hematogenous spread of causative bacteria from infections of organs other than the kidney itself, and Staphylococcus aureus accounted for $90 \%$ of the cases. However, there have been obvious changes and increases in ascending infections due to intrarenal reflux and urinary tract obstruction; Escherichia coli accounts for the majority of recent renal abscesses ${ }^{7)}$.

In the past, a renal abscess was treated with surgical drainage and antibiotics. However, in 1982, Finn et al. first performed PCD and successfully treated a renal abscess without surgery ${ }^{8}$. Luis recommended intravenous antibiotic treatment only for abscesses with a diameter of less than $3 \mathrm{~cm}$ but added invasive intervention for abscesses larger than $3 \mathrm{~cm}^{9}$.

The fornix of clayx is presumed to be the site of extravasation of urine in the kidney, and this forniceal leak accumulates around the kidney to form a urinoma in certain patients ${ }^{10}$. Subcapsular urinomas are usually observed in conditions of high renal intrapelvic pressure, such as ureteropelvic junction obstruction ${ }^{11)}$, megaureter, high-grade vesicoureteral reflux, posterior urethral valves, ureterocele, and urethral atresia ${ }^{12}$. Urinomas usually resolve when decompression procedures such as cutaneous pyelostomy or ureterostomy are performed in the kidney ${ }^{13)}$. In contrast, subcapsular fluid collection is often observed in patients with nephrotic syndrome, which reflects that the adhesion of the renal capsule to the renal parenchyma is not strong.

The pathogenesis of subcapsular renal abscess formation has not been fully elucidated; however, it is considered to be the same as that of renal subcapsular urinoma. Therefore, we believe that a solitary subcapsular abscess develops as an in situ lesion, rather than due to spread from an existing parenchymal abscess.

In case 1 , the DMSA scan did not reveal any renal damage despite repeated febrile UTIs; however, it was suspected that the growth of the right kidney could be retarded due to inflammatory restriction of the renal capsule and the aforementioned reflux. However, on the follow-up US after 3 years, the growth of both kidneys appeared normal: from $5.25 \mathrm{~cm}$ to $7.27 \mathrm{~cm}$ for the right kidney and from 5.33 $\mathrm{cm}$ to $6.90 \mathrm{~cm}$ for the left kidney. The previously noted high echo of the renal capsules was no longer observed (Fig. 5).
VCUG was not followed because there was no recurrence of UTI. In patient 2, the size of the abscess increased from $2.0 \mathrm{~cm}$ to $4.5 \mathrm{~cm}$ despite administration of antibiotics for 7 days; however, the kidney recovered almost completely through PCD.

With these cases, we can conclude that renal subcapsular abscess confined to the subcapsular space of the kidney appears to be caused by the direct reflux of infected urine to the capsule and can be successfully treated without considerable renal damage, if PCD is performed within an appropriate time.

\section{Patients consent}

This study was approved by the Institutional Review Board (IRB), and consent was waived due to the retrospective nature of the study (MED-EXP-21-505).

\section{Funding}

This research did not receive any specific grant from funding agencies in the public, commercial, or not-for-profit sectors.

\section{Conflicts of interest}

No potential conflict of interest relevant to this article was reported.

\section{ORCID}

Eun Ju Yoo https://orcid.org/0000-0001-5887-3478 Jae Hyuk Oh https://orcid.org/0000-0002-8827-7703 Hyun Joo Jung https://orcid.org/0000-0003-3614-1238

Su Jin Lee https://orcid.org/0000-0002-2096-6115 Ji Eun Park https://orcid.org/0000-0003-1305-0931 Ki Soo Pai https://orcid.org/0000-0003-0373-4336 


\section{References}

1. Bhat YR. Renal subcapsular abscess. Indian Pediatr 2007:44:5467.

2. Lee JH, Earm EJ, Han JS, Kim SW, Lee JS. Clinical features of renal and perirenal abscess. The Korean Journal of Nephrology 1990;9:357-63.

3. Choi SR, Kim CH, Kim SK, Oh JS. A case of multiple abscesses due to renal stone. Clin Exp Pediatr 1985;28:1245-9.

4. Sim JH, Yim HE, Yoo KH. Two cases of renal and perinephric abscesses in children. Korean Soc Pediatr Nephrol 2014;18:116-22.

5. Jeong JY, Park YS, Ham SY. A case of renal abscess in healthy child. J Korean Pediatr Soc 2000;43:1012-5.

6. Chi BH, Kim TH, Park SJ, Kim JY. A clinical survey of renal and perirenal abscess. Korean J Urogenit Tract Infect Inflamm 2009;4: 190-5.

7. Choi SY, Hwang DH, Yoon HS. A case of renal abscess in neonate.
J Korean Soc Neonatol 2005;12:200-5.

8. Finn DJ, Palestrant AM, DeWolf WC. Successful percutaneous management of renal abscess. J Urol 1982;127:425-6.

9. Seguias L, Srinivasan K, Mehta A. Pediatric renal abscess: a 10year single-center retrospective analysis. Hosp Pediatr 2012;2: 161-6.

10. Maitra NU, Conn IG. A unique case of spontaneous bilateral renal subcapsular urinoma secondary to high pressure chronic retention of urine. Urol Case Rep 2017;14:21-3.

11. Tsai JD, Huang FY, Lin CC, Tsai TC, Lee HC, Sheu JC, et al. Intermittent hydronephrosis secondary to ureteropelvic junction obstruction: clinical and imaging features. Pediatrics 2006;117:139-46.

12. Beetz R, Stein R, Rohatsch P, Brzezinska R, Thuroff JW. Acute perirenal extravasation of urine in an infant with non-refluxing megaureter. Pediatr Nephrol 2004;19:357-60.

13. Titton RL, Gervais DA, Hahn PF, Harisinghani MG, Arellano RS, Mueller PR. Urine leaks and urinomas: diagnosis and imagingguided intervention. Radiographics 2003;23:1133-47. 